\title{
Surgical Treatment of Brachycephalic Syndrome in Dogs
}

\author{
Erjavec $\mathbf{V}^{1, *}$, Lukanc $\mathbf{B}^{1}$
}

1. University of Ljubljana, Veterinary Faculty, Small Animal Clinic, Ljubljana, Slovenia

* Correspondence: Vladimira Erjavec; vladimira.erjavec@vf.uni-lj.si

Citation: Erjavec V, Lukanc B. Surgical treatment of brachycephalic syndrome in dogs. Proceedings of Socratic Lectures. 2021; 6: 23-29. https://doi.org/10.55295/PSL.2021.D. 004

Publisher's Note: UL ZF stays neutral with regard to jurisdictional claims in published maps and institutional affiliations.

Copyright: (c) 2021 by the authors Submitted for possible open access publication under the terms and conditions of the Creative Commons Attribution (CC BY) license (https://creativecommons.org/license s/by/4.0/).

\begin{abstract}
:
Brachycephalic breeds are becoming increasingly popular, despite various health problems due to a number of congenital anatomical features that differ from those of mesaticephalic breeds. Morphologic anomalies of the upper airway are primary (stenotic nostrils and elongated soft palate) and secondary due to increased airway resistance, negative intraluminal pressure and turbulent airflow. A total of 32 client-owned dogs of brachycephalic breeds were examined during the study period. The dogs were divided into 3 groups based on the severity of airway obstruction. All dogs underwent alaplasty and soft palate surgery, sacculectomy was performed in only 3 dogs. Based on the results of the questionnaire completed by owners before and 2 weeks after surgery, respiratory signs, gastrointestinal signs, exercise intolerance, and sleep disturbance improved in dogs in all groups of brachycephalic syndrome after surgery. It is important that veterinarians, as professionals, make the public aware of the potential problems associated with exaggerated brachycephalic characteristics. It is important that owners of a dog diagnosed with brachycephalic syndrome seek surgical treatment early in the affected dog's life. The owners of brachycephalic dogs should not perceive the clinical signs related to conformational inherited disorders as "normal" for the breed.
\end{abstract}

Keywords: Brachycephalic syndrome; Surgical treatment; Dogs; Staphylectomy; Alaplasty 


\section{Introduction}

Brachycephaly in dogs is the result of years of artificial selection for a short head shape. Originally, the brachycephalic phenotype was selected for fighting, but more recently brachycephalic breeds have been selected as pets with appealing appearance (Ekenstedt et al., 2020).

Brachycephalic syndrome (BS) has been identified in many brachycephalic breeds, including French and English bulldogs, pugs, Boston terriers, Shih-tzu, Pekingese, and Lhasa Apso (Trappler and Moore, 2011) and includes a variety of clinical signs such as loud upper respiratory sounds (stridor, stertor), sniffing, dyspnea, sleeping disorders, intolerance to exercise and heat, cyanosis, gastrointestinal signs, and in severe cases, fainting and occasionally death (Wykes, 1991; Poncet et al., 2005; Riecks et al., 2007; Roedler et al., 2013).

Morphologic anomalies of the upper airway are divided into primary and secondary. Primary anomalies include stenotic nostrils in which the nostril is deformed, and the opening is reduced to a vertical slit (Dupre and Heidereich, 2016), relative macroglossia (Ekenstedt et al., 2020), aberant or protruding nasal turbinates, narrow (naso)pharyngeal dimensions, an elongated soft palate, narrow laryngeal dimensions, and tracheal hypoplasia (Dupre and Heidereich, 2016; ter Haar and Sanches, 2017). As a result of increased airway resistance, negative intraluminal pressure, and turbulent airflow, secondary physical changes develop such as everted laryngeal saccules, everted tonsils, naso-pharyngeal collapse, and laryngeal, tracheal, and bronchial collapse (de Lorenzi et al., 2009), which may contribute to further obstruction (ter Haar and Sanches, 2017).

The initial diagnosis of brachycephalic syndrome is based on a combination of breed and clinical signs; further diagnostic imaging is recommended for therapeutic and prognostic reasons. Early diagnosis and surgical intervention of brachycephalic syndrome to improve airflow is important to prevent further deterioration and progression of secondary changes (Meola, 2013). Dogs with acute respiratory distress should be treated medically and preferably operated on at a later date after the edema and soft tissue inflammation have resolved. Treatment of affected dogs can be conservative (weight management and environmental modifications) or surgical. Several surgical techniques have been described to reduce the soft tissues at different levels.

The aim of our study was to evaluate the different anatomic changes in brachycephalic dogs and to assess the short-term outcome in dogs of different grades of dogs undergoing surgery for BS. In this article, we present our current surgical treatment of BS, which includes alaplasty and staphylectomy in all dogs and sacculectomy in selected cases.

\section{Methods}

A total of 32 dogs of brachycephalic breeds owned by clients were examined during the study period. After diagnosed with BS, 14 French bulldogs (FB), 9 pugs (P), 5 Boston terriers (BST), 3 English bulldogs (EB), and 1 Japanese Chin (JC) were included in the study.

A preoperative questionnaire on BS - related signs (respiratory signs, gastrointestinal signs, exercise intolerance, and sleep disturbance) was completed for each dog at initial presentation, and the same questionnaire was completed again two weeks after surgery. Diagnosis was based on clinical signs of upper airway obstruction, gastrointestinal clinical signs, and anatomic abnormalities, as described elsewhere (Dupre and Heidenreich, 2016). Formal written informed consent was obtained from the owner before the dogs participated in the study. All procedures complied with the relevant Slovenian governmental regulations (Animal Protection Act UL RS, 43/2007). The study 
was evaluated and approved by the Ethical Committee on Animal Research of the Veterinary Faculty, University of Ljubljana. The dogs were scheduled for general anaesthesia during which endoscopic examinations and surgical treatments were performed.

Data was analysed with commercial software (IBM SPSS 25, Chicago, Illinois, USA). Descriptive statistics were used to describe the basic features of the data (age, weight, body condition score (BCS)). The Shapiro Wilk test was performed to test whether the data were normally distributed. According to the results of normality tests, Kruskal Wallis test followed by multiple comparisons and Bonferroni adjustment was used to test for statistically significant differences in age, weight and BCS among groups of BOAS patients. Data are reported as medians and interquartile range (IQR -25 th to 75 th percentile). A value of $\mathrm{P}<0.05$ was considered significant.

\section{Results}

The dogs were classified into 3 grades according to the severity of obstruction at different anatomic levels, such as nasopharynx, oropharynx, laryngopharynx, and larynx, causing airway narrowing after soft palate surgery, as described elsewhere (Erjavec et al., 2021). Four dogs (1P, $3 \mathrm{FB}$ ) with no or minimal airway obstruction after nostril and soft palate surgery were classified as group 1, 16 dogs (3P, 3BST, 9FB, 1JC) with up to $50 \%$ airway obstruction after surgery were classified in group 2 , and $12 \operatorname{dogs}(5 \mathrm{P}, 3 \mathrm{~EB}$, 2BST, 2FB) with almost complete airway obstruction after surgery were classified in group 3. Baseline characteristics of the BOAS patients included in the individual groups are summarized in Table 1. We found no significant difference in age, weight and BCS among groups of BOAS patients. Evaluation of soft tissue anatomic changes revealed that all dogs (32/32) had stenotic nostrils and an overlong soft palate., everted tonsils were observed in 28/32 (87.5\%) dogs, $24 / 32$ (75\%) dogs had obstructed choanae, aberrant conchae were present in 15/32 (46.9\%) dogs, $23 / 32(71.9 \%)$ had redundant soft tissue (edematous mucosa) protruding through the choanae, and 31/32 (96.9\%) dogs had everted laryngeal saccules.

Table 1. Baseline characteristics in groups of brachycephalic obstructive airway syndrome patients.

\begin{tabular}{|l|c|c|c|}
\hline & Grade 1 & Grade 2 & Grade 3 \\
\hline Number & 4 & 16 & 12 \\
\hline $\begin{array}{l}\text { Sex } \\
\text { (female/male) }\end{array}$ & $3 / 1$ & $5 / 11$ & $5 / 7$ \\
\hline $\begin{array}{l}\text { Age (years) } \\
\text { Median }\end{array}$ & 2.41 & 2.88 & 1.75 \\
IQR & $1.08-4.75$ & $1.14-7.11$ & $1.44-7.90$ \\
\hline Weight (kg) & 9.78 & 10.20 & 9.68 \\
Median & $7.49-12.30$ & $7.78-12.53$ & $8.54-10.80$ \\
IQR & 3.0 & 3.0 & 3.0 \\
\hline BCS & $2.3-3.0$ & $3.0-4.0$ & $3.0-3.0$ \\
Median & IQR & & \\
\hline
\end{tabular}

BCS, body condition score; IQR, interquartile range (25th to 75 th percentile)

The dogs were intubated and the surgical procedure was performed under general anaesthesia with isoflurane (Isoflurine $1000 \mathrm{mg} / \mathrm{g}$, Chemical Iberica PV, Espana) in 100\% oxygen. For surgery, each dog was placed in a sternal position. Resection of the nostrils was performed with a horizontal wedge excision of the dorsomedial and caudal part of the alae with a No. 11 scalpel blade. Few simple interrupted sutures with the synthetic 
absorbable monofilament suture Glycomer 631 (Biosyn 4/0; Covidien, Dublin, Ireland) were placed to counteract the wedge margins (Figure 1).

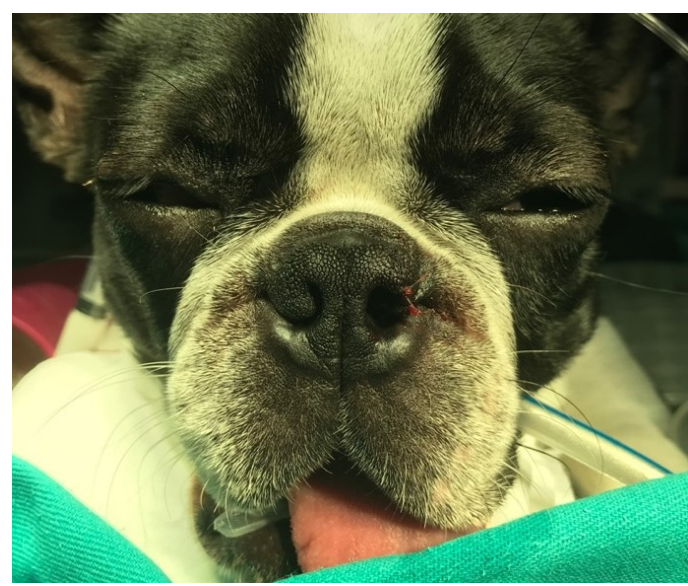

Figure 1. Nostrils in Boston terrier left side open nostril after the surgery, right nostril closed to a vertical slit.

Because many brachycephalic dogs have an excessively thick and long soft palate, a folded flap palatoplasty (FFP) (Findji and Dupre, 2008) was performed in all dogs. A portion of the oropharyngeal mucosa and underlying soft tissue was removed using a Surgitron Radiolase II (Ellman International, Inc., Hicksville, NY, USA) (Figure 2). In 3 of the 32 dogs, a sacculectomy was also performed. For sacculectomy, the dogs were extubated, and the saccules were grasped with forceps and cut with scissors. Minimal bleeding occurred.

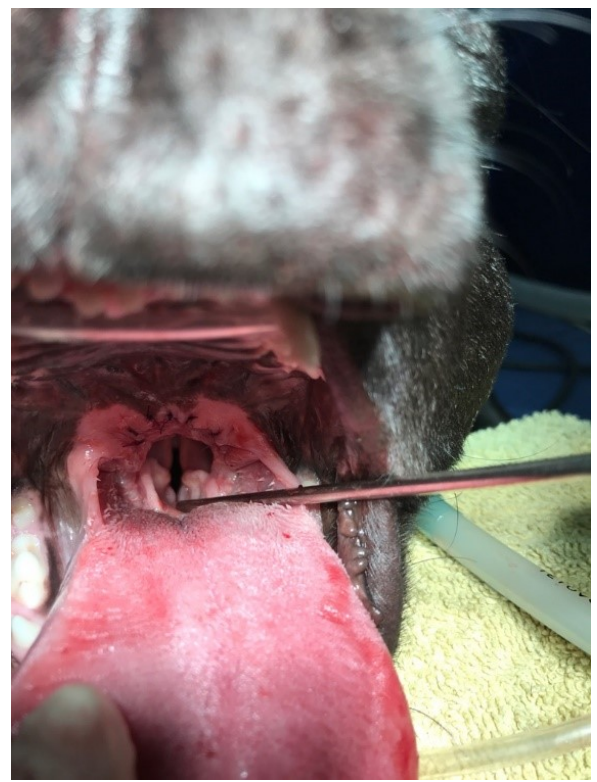

Figure 2. Soft palate after the folded flap palatoplasty surgery.

Based on the results of questionnaires completed by the owners, all clinical signs (respiratory signs, gastrointestinal signs, exercise intolerance, and sleep disturbance) in all groups of dogs improved two weeks after surgery. Before surgery, the most prominent clinical signs were respiratory problems and sleep disturbances; after surgery, sleep disturbances remained the most prominent clinical sign in all groups of dogs (Erjavec et al., 2021). 


\section{Discussion}

Surgery should be performed early to prevent further deterioration and possible airway collapse, but the optimal time for surgery has not yet been determined. It has been recommended to be performed after 6 months of age (Dupre and Heidenreich, 2016) and even at the time of spay or castration (Trappler and Moore, 2011). At Veterinary Faculty in Ljubljana, both procedures are not combined. We recommend operating on the BS first and evaluating the result at a later neutering. In dogs not operated at a young age and suffering from brachycephalic syndrome, which are middle-aged (Haimel and Dupre, 2015) and older than 10 years, surgery can still improve clinical signs and should be performed in all dogs suffering from brachycephalic syndrome. It is not entirely clear which part of the obstructed airway is most responsible for the clinical signs of BS (Ekenstedt et al. 2020). In our study, all dogs had some degree of stenosis of the nostrils and all dogs had an elongated soft palate. These findings are high compared to other studies (Poncet et al. 2005; Torrez and Hunt, 2006; Fasanela et al. 2010) but are consistent with the study by Seneviratne et al. (2020). Several techniques have been described for the correction of stenotic nostrils, no technique is more recommended than another, they all aim to allow more air into the nasal cavity. There was never a serious complication during this surgery; however, there is always a lot of bleeding during the procedure. The nasopharynx was narrowed in $75 \%$ of dogs due to mucosal folds and/or an aberrant caudal turbinate, and an aberrant caudal turbinate was present in $26.9 \%$ of dogs. Studies show partial regrowth of the removed turbinates, so the long-term effects of turbinectomy need to be further investigated (Schuenemann and Oechtering, 2014). All dogs underwent FFP to shorten an excessively long and thick soft palate. The soft palate is shortened by retracting the caudal edge of the soft palate and folding it onto itself. With FFP, the surgical site is moved rostrally and possible edema of the wound may be less obstructive to the airway. Although it has been reported that up to $28 \%$ of dogs require a tracheostomy after surgical treatment of BS (Dupre and Heidereich, 2016), none of the dogs in our study required this. We hypothesise that the effect of this surgery is due to both the opening of the nasopharynx and the removal of the rima glottidis obstruction.

In many brachycephalic dogs, the tongue is 10 times denser than in mesaticephalic dogs (Jones et al. 2020), thick and excessively long (relative macroglossia), and displaces the soft palate further dorsally, which, together with hypertrophy of the nasopharyngeal mucosa (Ekenstedt et al., 2020), results in airway obstruction during nasal breathing. However, the tongue volume in pugs is smaller than in French and English bulldogs (Siedenburg and Dupre, 2021). In our study, we observed that the tongue in pugs is relatively longer than in other brachycephalic dogs and that it is not as thick and therefore does not contribute to breathing problems. To date, no surgical techniques to reduce the size of the tongue have been successful in treating BS.

Everted tonsils used to be treated with tonsillectomy, but this is no longer recommended because minimal or no effect was observed after tonsil removal (Poncet et al., 2005; Poncet et al., 2006). We have so far never removed tonsils for the treatment of BS. Laryngeal saccules can be surgically removed using a variety of instruments or devices, but the complication rate after removal is high, so routine removal is not recommended (Huges et al., 2018). At the Veterinary faculty we have removed them only when everted saccules contributed significantly to obstruction and when further anaesthesia would pose a high risk to the patient. The trachea may be congenitally hypo-plastic, with tracheal cartilages that touch or overlap. While this may exacerbate respiratory clinical signs, it is not considered a major cause BS (Ekenstedt et al., 2020) and does not affect 
postoperative outcomes (Riecks et al., 2007). In addition, this condition cannot be treated surgically. Collapsed trachea and bronchi are the result of chronically increased airway pressure (ter Haar and Sanches, 2017) and, according to our observations, occur mainly in pugs.

Possible complications during surgery from BS and postoperatively include bleeding, vomiting, hyperthermia, aspiration pneumonia, respiratory problems due to soft tissue edema, or airway obstruction by saliva, mucus, and/or blood. Close patient monitoring is essential. Professional help must be sought immediately if there is retching, vomiting, or possible respiratory problems. Several techniques have been described for the correction of narrowed nostrils. No one technique is more recommended than another; they all aim to allow more air into the nasal cavity. Limited exercise (walks on a harness, no playing with other dogs) is recommended for at least one week after surgery to avoid the complications mentioned above.

\section{Conclusions}

Our study shows that respiratory signs, gastrointestinal signs, exercise intolerance, and sleep disturbance improved in dogs in all groups of BS after surgery. Nevertheless, surgery is recommended at an early age when BS is diagnosed because the more severe secondary physical changes may develop as a result of increased airway resistance, negative intraluminal pressure, and turbulent airflow. Surgical treatment of BS usually involves alaplasty and palatoplasty, and occasionally a sacculectomy may be performed.

Funding: This research was supported by Slovenian Research Agency (research program No. P4-0053). Institutional Review Board Statement: All procedures complied with the relevant Slovenian governmental regulations (Animal Protection Act UL RS, 43/2007).

Conflicts of Interest: The authors declare no conflict of interest.

Acknowledgements: The authors thank Alenka Nemec Svete for her valuable suggestions and professional help in designing and finalising this paper.

\section{References}

1. De Lorenzi D, Bertoncello D, Drigo M. Bronchial abnormalities found in a consecutive series of 40 brachycephalic dogs. J Am Vet Med Assoc. 2009; 235: 835-40. DOI: 10.2460/javma.235.7.835.

2. $\quad$ Dupre G, Heidereich D. Brachycephalic Syndrome. Vet Clin Small Anim. 2016; 46: 691-707 http://dx.doi.org/10.1016/j.cvsm.2016.02.002

3. Ekenstedt KJ, Crosse KR, Risselada M. Canine Brachycephaly: Anatomy, Pathology, Genetics and Welfare. J Comp Pathol. 2020; 176: 109-115. DOI: 10.1016/j.jcpa.2020.02.008.

4. Erjavec V, Vovk T, Svete A. Evaluation of oxidative stress parameters in dogs with brachycephalic obstructive airway syndrome before and after surgery. J Vet Res. 2021; 65: 201-208. https://doi.org/10.2478/jvetres-2021-0027.

5. Findji L, Dupré G. Folded flap palatoplasty for treatment of elongated soft palates in 55 dogs. Eur J Companion Anim Pract. 2008; 19: 125-132.

6. Haimel G, Dupré G. Brachycephalic airway syndrome: a comparative study between pugs and French bulldogs. J Small Anim Pract. 2015; 56: 714-9. DOI: 10.1111/jsap.12408.

7. Fasanella FJ, Shivley JM, Wardlaw JL, et al. Brachycephalic airway obstructive syndrome in dogs: 90 cases (1991-2008). J Am Vet Med Assoc. 2010; 237: 1048-51. DOI: 10.2460/javma.237.9.1048

8. Haar G.ter, Sanchez RF. Brachycephaly-related diseases. In: Small Dogs - Big Problems, Veterinary Focus. 2017; 27: 15-22.

9. Hughes JR, Kaye BM, Beswick AR, Ter Haar G. Complications following laryngeal sacculectomy in brachycephalic dogs. J Small Anim Pract. 2018; 59: 16-21. doi: 10.1111/jsap.12763.

10. Jones BA, Stanley BJ, Nelson NC. The impact of tongue dimension on air volume in brachycephalic dogs. Vet Surg. 2020; 49 :512-520. DOI: 10.1111/vsu.13302.

11. Meola SD. Brachycephalic airway syndrome. Top Companion Anim Med. 2013; 28: 91-96. DOI: 10.1053/j.tcam.2013.06.004

12. Poncet CM, Dupré G, Freiche VG, et al. Prevalence of gastrointestinal tract lesions in 73 brachycephalic dogs with upper respiratory syndrome. J Small Anim Pract. 2005; 46: 273-9. DOI: 10.1111/j.1748-5827.2005.tb00320.x.

13. Poncet CM, Dupre GP, Freiche VG, Bouvy BM. Long-term results of upper respiratory syndrome surgery and gastrointestinal tract medical treatment in 51 brachycephalic dogs. J Small Anim Pract 2006; 47: 137-42.

DOI: $10.1111 /$ j.1748-5827.2006.00057.x 
14. Riecks TW, Birchard SJ, Stephens JA. Surgical correction of brachycephalic syndrome in dogs: 62 cases (1991-2004). J Am Vet Med Assoc. 2007; 230: 1324-1328. DOI: 10.2460/javma.230.9.1324

15. Roedler FS, Pohl S, Oechtering GU. How does severe brachycephaly affect dog's lives? Results of a structured preoperative owner questionnaire. Vet J. 2013; 198: 606-610. DOI: 10.1016/j.tvj1.2013.09.00

16. Schuenemann R, Oechtering G. Inside the brachycephalic nose: conchal regrowth and mucosal contact points after laser-asisted turbinectomy. J Am Anim Hosp Assoc. 2014; 50: 237-46. DOI: 10.5326/JAAHA-MS-6086

17. Siedenburg JS, Dupré G. Tongue and Upper Airway Dimensions: A Comparative Study between Three Popular Brachycephalic Breeds. Animals (Basel). 2021; 11:662. doi: 10.3390/ani11030662.

18. Torrez CV, Hunt GB. Results of surgical correction of abnormalities associated with brachycephalic airway obstruction syndrome in dogs in Australia. J Small Anim Pract. 2006; 47:150-4. DOI: 10.1111/j.1748-5827.2006.00059.x

19. Trappler M, Moore K. Canine brachycephalic airway syndrome: surgical management. Compend Contin Educ Vet. 2011; 33:E1-7. PMID: 21870354.

20. Wykes PM. Brachycephalic airway obstructive syndrome. Probl Vet Med. 1991; 3: 188-97. PMID: 1802247. 